\title{
Secondary resistance to imatinib in patients with gastrointestinal stromal tumors through an acquired KIT exon 17 mutation
}

\author{
C.M. WANG ${ }^{1}$, H. FU ${ }^{1}$, G.F. ZHAO ${ }^{1}$, X.Y. ZHOU ${ }^{2}$, C.Y. DU ${ }^{1}$, R.Z. DONG ${ }^{1}$, Y. ZHOU ${ }^{1}$ and Y.Q. SHI ${ }^{1}$ \\ ${ }^{1}$ Abdominal Cancer Institute, ${ }^{2}$ Pathology Cancer Institute, Cancer Hospital/Cancer Institute, \\ Department of Oncology, Shanghai Medical College, Fudan University, Shanghai 200032, P.R. China
}

Received November 7, 2008; Accepted January 21, 2009

DOI: 10.3892/mmr_00000121

\begin{abstract}
Although imatinib has demonstrated a potent effect on advanced gastrointestinal stromal tumors (GISTs) and has improved the survival of GIST patients, with its prolonged use imatinib resistance is becoming an increasing clinical problem. Mechanisms of secondary resistance are still under investigation. Our study aimed to determine the mechanism of acquired resistance to imatinib in GISTs. Using bidirectional PCR DNA sequencing, we sequenced exons 9, 11, 13 and 17 of the KIT gene and exons 12 and 18 of the PDGFRA gene in secondary resistant lesions obtained from 18 GIST patients after treatment with imatinib. Fourteen of 18 cases carried activating mutations in the KIT gene, with a mutation encoding the juxtamembrane domain present in exon 11 in 12 cases, in exon 13 in 1 case, and in exon 9 in 1 case. In 4 of 10 imatinib-resistant patients, an identical novel missense mutation $(\mathrm{T} 2467 \mathrm{G})$ was found in exon 17 , resulting in a substitution of tyrosine by aspartic acid at codon 823 (Y823D). In conclusion, the exon 17 missense mutation $\mathrm{T} 2467 \mathrm{G}$ in the tyrosine kinase domain of the KIT gene is correlated with imatinib resistance.
\end{abstract}

\section{Introduction}

Gastrointestinal stromal tumors (GISTs) are the most common mesenchymal tumors of the gastrointestinal tract. Activating oncogenic mutations in the KIT (stem cell factor receptor) gene or in the platelet derived growth factor receptor $\alpha$ (PDGFRA) gene are related to the pathogenesis of this malignancy. Detection of the KIT tyrosine kinase receptor and the development of imatinib, which blocks the activity of this receptor, have changed the treatment and outlook on this malignancy, with imatinib significantly improving the survival of patients

Correspondence to: Dr Ying Qiang Shi, Abdominal Cancer Institute, Cancer Hospital/Cancer Institute, Department of Oncology, Shanghai Medical College, Fudan University, 270 Dong'an Road, Shanghai 200032, P.R. China

E-mail: shiyingqiang@csco.cn

Key words: gastrointestinal stromal tumor, imatinib mesylate, acquired resistance, KIT gene with advanced GIST. However, with longer durations of imatinib treatment, the number of patients who develop progressive disease increases continuously. It is generally held that all GIST patients treated with imatinib for advanced disease will inevitably develop progressive disease. The median progression-free survival time is approximately 24 months (1). There appear to be two forms of resistance: early resistance, which is defined as progression occurring in patients who never showed any response to imatinib or as progression encountered within 3 months of the initiation of imatinib, and late resistance, which is defined as progression occurring in patients who initially responded to treatment or who had a progression-free survival interval exceeding 3 months (2). Several different mechanisms of GIST resistance to imatinib have been identified: i) acquired KIT or PDGFRA mutations resulting in intrinsic target resistance; ii) genomic amplification leading to target overexpression; iii) alternate oncoprotein activation supplanting target expression; and iv) functional resistance caused by heterogeneous mechanisms, including mutations outside the juxtamembrane hotspot region $(3,4)$. Nevertheless, the precise mechanisms of secondary resistance to imatinib are still under investigation. In the present study, we aimed to explore the nature of secondary KIT mutations occurring in patients undergoing imatinib therapy, as well as the mechanisms of secondary resistance to imatinib.

\section{Materials and methods}

Patients. From June 2000 to January 2008, 18 patients (12 men and 6 women; median age 43 years; range 28-68 years) diagnosed with GIST were treated with imatinib and were closely followed at Fudan University Affiliated Cancer Hospital, P.R. China. Detailed drug treatment records were maintained. Clinical response was evaluated according to the Response Evaluation Criteria in Solid Tumors (RECIST) (5). Four weeks after the initiation of imatinib therapy, magnetic resonance tomography or a computer-assisted tomography scan was used to detect early tumor progression. Thereafter, reassessment of response was conducted every 3 months. Imatinib treatment was halted $24 \mathrm{~h}$ before surgical procedures in accordance with standard care. Tumor tissue specimens obtained during surgery from all patients undergoing imatinib therapy and formalin-fixed tissues acquired from patients prior to treatment were used for molecular analysis. Common hotspots for primary and secondary mutations in exons $9,11,13$ 
Table I. Primer sequences.

\begin{tabular}{|c|c|c|c|c|}
\hline No. & Type & Primer sequence & Exon & Region in $K I T / P D G F R A$ \\
\hline 1 & Genomic & $\begin{array}{l}\text { F: 5-ATTTATTTTCCTAGAGTAAGCCAGGG-3 } \\
\text { R: 5-ATCATGACTGATATGGTAGACAGAGC-3 }\end{array}$ & 9 & KIT \\
\hline 2 & Genomic & $\begin{array}{l}\text { F: 5-CTCCAGAGTGCTCTAATGACTGAGAC-3 } \\
\text { R: 5-GTCACTGTTATGTGTACCCAAAAAGG-3 }\end{array}$ & 11 & KIT \\
\hline 3 & Genomic & $\begin{array}{l}\text { F: 5-GCTTGACATCAGTTTGCCAGTTGTGC-3 } \\
\text { R: 5-GACAGACAATAAAAGGCAGCTTGGAC-3 }\end{array}$ & 13 & KIT \\
\hline 4 & Genomic & $\begin{array}{l}\text { F: 5-CTTTTCTCСТССААССТАATAGTG-3 } \\
\text { R: 5-TTGAAACTAAAAATCCTTTGCAGGAC-3 }\end{array}$ & 17 & KIT \\
\hline 5 & Genomic & $\begin{array}{l}\text { F: 5-GTGCACTGGGACTTTGGTAATTC-3 } \\
\text { R: 5-GTGCAAGGGAAAAGGGAGTCTTG-3 }\end{array}$ & 12 & PDGFRA \\
\hline 6 & Genomic & $\begin{array}{l}\text { F: 5-GCTATTCAGCTACAGATGGCTTG-3 } \\
\text { R: 5-AAGTGAAGGAGGATGAGCCTGAC-3 }\end{array}$ & 18 & PDGFRA \\
\hline
\end{tabular}

F, forward; R, reverse.

and 17 of the KIT gene as well as in exons 12 and 18 of the PDGFRA gene were analyzed. The study was carried out under the guidelines of the affiliated institutions, and written informed patient consent was obtained. The last date of follow-up was January 31, 2008.

Histopathology and immunohistochemistry. The histomorphologic subtype was classified as spindle cell type, epithelioid cell type and mixed type, as previously described (6). Diagnosis of GIST was confirmed by immunohistochemical analysis using antibodies against CD117 (KIT receptor), CD34, smooth muscle actin (SMA), bcl-2, $\alpha$-actin, desmin, S-100 protein, vimentin (all from Dako, Hamburg, Germany), PDGFRA (Santa Cruz Biotechnology, Santa Cruz, CA) and Ki-67 (MIB-1, Dianova, Hamburg, Germany) as previously described $(7,8)$.

Sequence analysis in KIT and PDGFRA genes. The fresh samples were flash frozen in liquid nitrogen at the time of surgical resection or biopsy and maintained at $-80^{\circ} \mathrm{C}$, and genomic DNA was extracted following standard procedures. Total DNA was extracted after pre-treatment with proteinase $\mathrm{K}$ and absorption on silica gel membranes (Qiagen, Hilden, Germany). After the estimation of DNA concentration by agarose gel electrophoresis, the relevant exons were amplified with intronic primers as previously described (9) (Table I). The PCR products were purified using MicroSpin columns (Amersham Biosciences, Freiburg, Germany). Bidirectional DNA sequencing of the entire exons and their corresponding exon-intron boundaries was carried out with the Big Dye Terminator Cycle Sequencing Ready Reaction Kit (Applied Biosystems, Weiterstadt, Germany). Cycle sequencing products were precipitated with $3 \mathrm{~mol} / \mathrm{l}$ sodium acetate and analyzed on an ABI PRISM 310 Capillary Electrophoresis System (Applied Biosystems). All sequence changes were confirmed by an independent PCR amplification and by sequencing to exclude PCR artifacts. The identity of the amplicon sequences was confirmed by a database search.

\section{Results}

Median follow-up time from the initial diagnosis to the final evaluation was 39 months (range 11-85 months). The median duration of response to imatinib treatment (progression-free survival; Table II) was 11 months (range 0-45 months). The primary tumors were predominantly located in the stomach $(6 / 18)$, while 5 tumors arose in the small intestine and 3 in the rectum or colon. Four were extragastrointestinal stromal tumors. Tumor size ranged from 4 to $20 \mathrm{~cm}$. Initially, patients were orally administered $400 \mathrm{mg}$ imatinib once daily. When 7 of the patients became insensitive to imatinib, an increased dose of $600 \mathrm{mg}$ once daily was administered to 2 patients, while the other 5 received an increased dose of $400 \mathrm{mg}$ bid per day. Imatinib treatment was interrupted in $6 / 18$ patients for periods of 2-11 months; 4 for financial reasons and 2 due to adverse drug effects. Four patients still showed sensitivity to imatinib after treatment was resumed, while cases 9 and 10 exhibited primary tumor progression. The tumors of 8 patients who developed secondary tumor progression while undergoing imatinib treatment were resected palliatively after 5-45 months of response (median 12 months). Eight patients who were responsive to imatinib received resection after 8-25 months of treatment (median 11.5 months). Two patients who were classified as having primary resistance to imatinib received palliative resection immediately after developing disease progression. The clinical and pathologic data for all the cases are listed in Table II.

Histopathologic and immunohistochemical findings. Ten tumors were of spindle cell type, 2 were of epithelioid cell type and 6 GISTs displayed a mixed morphology. In two cases, different morphologic subtypes were noted between the primary tumor and metastases. Both showed spindle cell type at the primary site but mixed type in the metastases. The immunohistochemical examination revealed that $16 / 18$ (88.9\%) of the tumors exhibited positive staining for CD117, while 13/18 (72.2\%) exhibited positive staining for CD34 and 


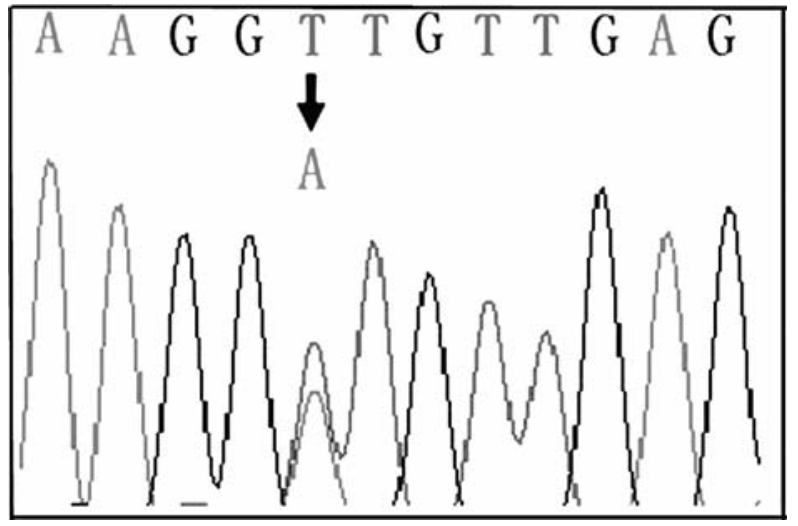

Figure 1. Case 3 primary mutation prior to imatinib treatment. The mutation $(\mathrm{C} 1676 \mathrm{~T} \rightarrow \mathrm{A})$ was detected in exon 11 of the KIT gene, and always led to a substitution of praline by aspartic acid in codon 559 (P559D).

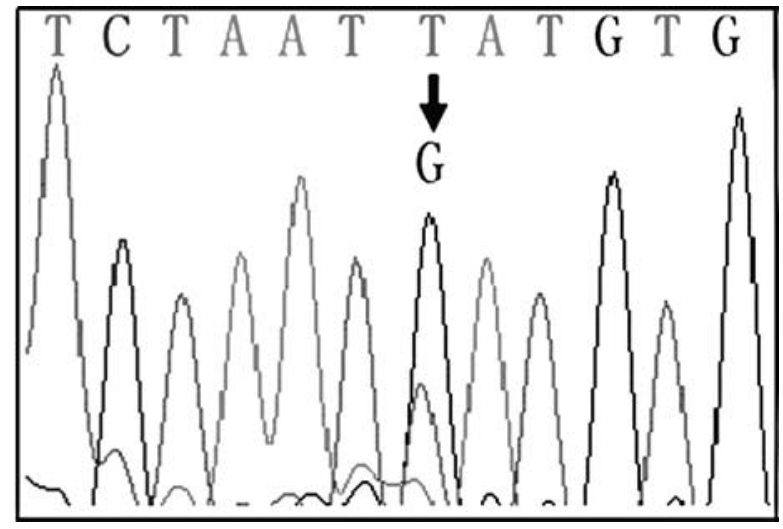

Figure 2. Case 3 secondary mutation after imatinib treatment. The mutation $(2467 \mathrm{~T} \rightarrow \mathrm{G})$ was detected in exon 17 of the KIT gene, and always led to a substitution of tyrosine by aspartic acid in codon 823 (Y823D).

Table II. Clinical and pathologic data of patients with GISTs under imatinib treatment.

\begin{tabular}{|c|c|c|c|c|c|c|c|c|c|}
\hline $\begin{array}{l}\text { Case } \\
\text { no. }\end{array}$ & $\begin{array}{l}\text { Age/ } \\
\text { Gender }\end{array}$ & Primary location & $\begin{array}{l}\mathrm{MD} \\
(\mathrm{cm})\end{array}$ & Metastases & $\begin{array}{c}\text { Type of } \\
\text { response (PFS) }\end{array}$ & CD117 & CD34 & $\begin{array}{c}\text { OS } \\
\text { (months) }\end{array}$ & Outcome \\
\hline 1 & $37 / \mathrm{M}$ & Small bowel & 12.3 & Primary site & $\mathrm{SP}(35)$ & + & - & 80 & DOD \\
\hline 2 & $38 / \mathrm{M}$ & Small bowel & 12.0 & Rectal & SP (38) & + & - & 83 & DOD \\
\hline 3 & $57 / \mathrm{F}$ & Small bowel & 20.0 & Peritoneum & SP (43) & + & + & 56 & DOD \\
\hline 4 & $47 / \mathrm{F}$ & Small bowel & 16.0 & Peritoneum & SP (28) & + & + & 53 & AWD \\
\hline 5 & 43/M & Colon & 13.0 & Peritoneum & SP (9) & - & - & 37 & AWD \\
\hline 6 & $27 / \mathrm{M}$ & Extragastrointestinal & NDA & Liver + peritoneum & $\mathrm{SP}(12)$ & ++ & - & 39 & AWD \\
\hline 7 & 39/M & Stomach & 5.0 & Liver + abdominal & SP (37) & ++ & ++ & 66 & AWD \\
\hline 8 & $42 / \mathrm{M}$ & Small bowel & 10.0 & Liver & $\mathrm{SP}$ (5) & +++ & +++ & 54 & AWD \\
\hline 9 & $68 / \mathrm{F}$ & Extragastrointestinal & 15.0 & Liver + lung & PP (0) & + & + & 30 & DOD \\
\hline 10 & $51 / \mathrm{M}$ & Stomach & 20.0 & Extragastrointestinal & $\mathrm{PP} \quad(0)$ & - & - & 12 & AWD \\
\hline 11 & $35 / \mathrm{M}$ & Stomach & 14.0 & Peri-spleen & SD (3) & + & + & 31 & AWD \\
\hline 12 & $41 / \mathrm{M}$ & Small bowel + liver & 8.5 & None & SD (2) & + & - & 4 & AWD \\
\hline 13 & $51 / \mathrm{M}$ & Stomach & 19.0 & Peri-pancreas + liver & SD (6) & + & + & 20 & AWD \\
\hline 14 & $53 / \mathrm{F}$ & Small bowel & 8.0 & Extragastrointestinal & $\mathrm{SD}(12)$ & + & - & 81 & AWD \\
\hline 15 & $58 / \mathrm{F}$ & Rectum & 4.0 & Liver + lung & $\mathrm{SD}(11)$ & + & + & 33 & AWD \\
\hline 16 & $68 / \mathrm{M}$ & Extragastrointestinal & 12.0 & Primary site & $\mathrm{SD}(11)$ & + & + & 48 & AWD \\
\hline 17 & $50 / \mathrm{M}$ & Stomach & 6.0 & Liver + abdominal & PR (13) & + & + & 16 & AWD \\
\hline 18 & $59 / \mathrm{M}$ & Colon & 6.5 & Colon + peritoneum & SD (6) & + & + & 39 & AWD \\
\hline
\end{tabular}

MD, maximum diameter; PFS, progression-free survival; OS, overall survival; NDA, no data available; SP, secondary progression; PP, primary progression; SD, stable disease; PR, partial remission; DOD, dead of disease; AWD, alive with disease.

some for SMA, but not for bcl-2, $\alpha$-actin, desmin, vimentin and S-100 protein. In 2/18 primary tumors, the KIT receptor had at least weak expression before treatment. Furthermore, strong re-expression of the KIT receptor was observed in progressive lesions.

Primary KIT mutations (Table III). In 14/18 cases carrying activating mutations in the KIT gene, 12 (66.7\%), including 5 of imatinib secondary resistance, 6 achieving stable disease and 1 showing partial remission, were found to possess mutations in exon 11 encoding the juxtamembrane domain; 1 patient was found to have a mutation in exon 13 , and 1 had a mutation in exon 9. These mutations included 9 deletions of 6-27 bp (such as 1651-1668del, 1670-1675del and 1693-1719del) between codons 551 and 576 as well as 3 point mutations $(1650 \mathrm{~A} \rightarrow \mathrm{G}, 1669 \mathrm{~T} \rightarrow \mathrm{A}$ and $1676 \mathrm{~T} \rightarrow \mathrm{A})$. The mutation in exon 13 $(1924 \mathrm{~A} \rightarrow \mathrm{G})$ was detected in 1 patient (case 12) $(7.1 \%)$ who achieved stable disease, always leading to a substitution of lysine to glutamic acid (K642E). One patient (7.1\%) that exhibited secondary resistance had mutations in exon 9, which displayed tandem duplication in codons 502 and 503. In 2 primary resistant patients $(11.1 \%)$ and 2 secondary resistant patients $(11.1 \%)$, wild-type sequences were found in all the examined exons of KIT and PDGFRA (wild-type GISTs). Among the GISTs, no primary mutations were found in exon 17 of KIT or in exons 12 and 18 of PDGFRA. 
Table III. Primary and secondary KIT and PDGFRA mutations of 18 GIST cases undergoing imatinib treatment.

\begin{tabular}{|c|c|c|c|c|c|c|}
\hline \multirow[b]{2}{*}{ Case no. } & \multicolumn{4}{|c|}{$K I T$ gene } & \multirow{2}{*}{$\frac{P D G F R A \text { gene }}{\text { Exon } 12 \text { and } 18}$} & \multirow[b]{2}{*}{ Secondary mutation } \\
\hline & Exon 9 & Exon 11 & Exon 13 & Exon 17 & & \\
\hline \multicolumn{7}{|l|}{ Case 1} \\
\hline Before imatinib & Normal & $1650 \mathrm{~A} \rightarrow \mathrm{G}$ & Normal & Normal & Normal & \\
\hline After & Normal & $1650 \mathrm{~A} \rightarrow \mathrm{G}$ & Normal & $2467 \mathrm{~T} \rightarrow \mathrm{G}$ & Normal & Yes \\
\hline \multicolumn{7}{|l|}{ Case 2} \\
\hline Before imatinib & Normal & del551-556 & Normal & Normal & Normal & \\
\hline After & Normal & del551-556 & Normal & $2467 \mathrm{~T} \rightarrow \mathrm{G}$ & Normal & Yes \\
\hline \multicolumn{7}{|l|}{ Case 3} \\
\hline Before imatinib & Normal & $1676 \mathrm{~T} \rightarrow \mathrm{A}$ & Normal & Normal & Normal & \\
\hline After & Normal & $1676 \mathrm{~T} \rightarrow \mathrm{A}$ & Normal & $2467 \mathrm{~T} \rightarrow \mathrm{G}$ & Normal & Yes \\
\hline \multicolumn{7}{|l|}{ Case 4} \\
\hline Before imatinib & Normal & del565-573 & Normal & Normal & Normal & \\
\hline After & Normal & del565-573 & Normal & $2467 \mathrm{~T} \rightarrow \mathrm{G}$ & Normal & Yes \\
\hline \multicolumn{7}{|l|}{ Case 5} \\
\hline Before imatinib & Normal & Normal & Normal & Normal & Normal & \\
\hline After & Normal & Normal & Normal & Normal & Normal & None \\
\hline \multicolumn{7}{|l|}{ Case 6} \\
\hline Before imatinib & Normal & Normal & Normal & Normal & Normal & \\
\hline After & Normal & Normal & Normal & Normal & Normal & None \\
\hline \multicolumn{7}{|l|}{ Case 7} \\
\hline Before imatinib & dup $502+503$ & Normal & Normal & Normal & Normal & \\
\hline After & dup502+503 & Normal & Normal & Normal & Normal & None \\
\hline \multicolumn{7}{|l|}{ Case 8} \\
\hline Before imatinib & Normal & del556-559 & Normal & Normal & Normal & \\
\hline After & Normal & del556-559 & Normal & Normal & Normal & None \\
\hline \multicolumn{7}{|l|}{ Case 9} \\
\hline Before imatinib & Normal & Normal & Normal & Normal & Normal & \\
\hline After & Normal & Normal & Normal & Normal & Normal & None \\
\hline \multicolumn{7}{|l|}{ Case 10} \\
\hline Before imatinib & Normal & Normal & Normal & Normal & Normal & \\
\hline After & Normal & Normal & Normal & Normal & Normal & None \\
\hline \multicolumn{7}{|l|}{ Case 11} \\
\hline Before imatinib & Normal & del558-559 & Normal & Normal & Normal & \\
\hline After & Normal & del558-559 & Normal & Normal & Normal & None \\
\hline \multicolumn{7}{|l|}{ Case 12} \\
\hline Before imatinib & Normal & Normal & $1924 \mathrm{~A} \rightarrow \mathrm{G}$ & Normal & Normal & \\
\hline After & Normal & Normal & $1924 \mathrm{~A} \rightarrow \mathrm{G}$ & Normal & Normal & None \\
\hline \multicolumn{7}{|l|}{ Case 13} \\
\hline Before imatinib & Normal & $1669 \mathrm{~T} \rightarrow \mathrm{A}$ & Normal & Normal & Normal & \\
\hline After & Normal & $1669 \mathrm{~T} \rightarrow \mathrm{A}$ & Normal & Normal & Normal & None \\
\hline \multicolumn{7}{|l|}{ Case 14} \\
\hline Before imatinib & Normal & del557-558 & Normal & Normal & Normal & \\
\hline After & Normal & del557-558 & Normal & Normal & Normal & None \\
\hline Case 15 & & & & & & \\
\hline Before imatinib & Normal & del557-561 & Normal & Normal & Normal & \\
\hline After & Normal & del557-561 & Normal & Normal & Normal & None \\
\hline Case 16 & & & & & & \\
\hline Before imatinib & Normal & del552-553 & Normal & Normal & Normal & \\
\hline After & Normal & del552-553 & Normal & Normal & Normal & None \\
\hline Case 17 & & & & & & \\
\hline Before imatinib & Normal & del551-554 & Normal & Normal & Normal & \\
\hline After & Normal & del551-554 & Normal & Normal & Normal & None \\
\hline Case 18 & & & & & & \\
\hline Before imatinib & Normal & del576 & Normal & Normal & Normal & \\
\hline After & Normal & del576 & Normal & Normal & Normal & None \\
\hline
\end{tabular}

Cases 5 and 6 after imatinib treatment, tumor stable. Cases 7, 8 and 9, before imatinib treatment. 
Secondary mutations in KIT with imatinib treatment (Table III). Secondary KIT mutations were identified in 4/10 patients (40\%), with all 4 patients developing one identical type of secondary mutation in the tyrosine kinase domain of exon 17 in the KIT gene $(2467 \mathrm{~T} \rightarrow \mathrm{G})$, always leading to a substitution of tyrosine by aspartic acid (Y823D). Of note, all 4 of the patients with primary mutations detected in exon 11 exhibited acquired resistance to imatinib. The secondary mutations detected were missense mutations occurring in exon 17. None of the mutations were found in exons 9, 11 and 13 of the KIT gene, nor in exons 12 and 18 of the PDGFRA gene, possibly as a result of the limited sample size. Two primary progressive diseases and 8 patients with stable disease or partial remission were not found to carry any secondary mutations.

\section{Discussion}

Since the first case report in 2001 on its successful application (10), imatinib mesylate has been established as a firstline therapy for unresectable, metastatic and/or recurrent GISTs (11). Although imatinib has revolutionized the treatment of advanced GISTs, with its prolonged administration clinical resistance to this drug has proven to be a significant problem. Recent reports have shown various degrees of secondary mutations in the kinase domain of the target genes concomitant with re-activation of the corresponding kinase, even in the presence of imatinib (12-14). KIT and PDGFRA are type III tyrosine kinases in which the kinase domain splits into domains I and II. The former is mainly composed of exons 13 and 14, which form the ATP-binding domain, and the latter is composed of exons 16 and 17, which form an activation loop. Mutations in the activation loop are rather variable compared to the ATP-binding domain. Antonescu et al (15) found that the activation loop (A-loop) of the KIT kinase, which includes Y823, a pseudosubstrate of the KIT kinase, is another major site of oncogenic mutation. During KIT kinase activation, Y823 becomes phosphorylated. This seems to stabilize the open active conformation of the A-loop, presumably by strong negative electrostatic interactions of the phosphate residue. Oncogenic activation loop mutations such as Y823D, which mimic Y823 phosphorylation, thus stabilize the active conformation of the A-loop. The structure of the KIT-imatinib complex revealed that, similar to BCR-ABL, imatinib binds the inactive conformation of the kinase although the KIT-imatinib complex deviates somewhat from the autoinhibited inactive KIT kinase conformation. It is therefore not surprising that A-loop mutations are generally not inhibited by imatinib, although there seem to be exceptions (16). Our findings are in agreement with these predictions. In our study, the second mutation site, Tyr 823 (located in the A-loop), was found to be substituted by aspartic acid in 4 patients with resistant tumors.

In the present study, $40 \%$ of the patients with imatinibresistant GISTs had secondary mutations. Although not statistically significant, the frequency of secondary KIT mutations was higher in tumors with a primary KIT exon 11 mutation than in those with primary KIT exon 9 or 13 mutations. As shown by several groups $(17,18)$, the location of the primary KIT or PDGFRA mutation directly predicts clinical response to imatinib. When the inhibitory effect of imatinib, depending on the underlying activating mutation, is low, the larger amount of remaining tumor might lead to an increased risk of secondary mutation. However, our results show that tumors with an underlying primary KIT mutation in exon 11, known to be the subgroup with better response rates than other mutational subtypes, are at a higher risk of developing secondary mutations than those with a KIT exon 9 or exon 13 mutation. It has been reported that the cessation of imatinib or administration of subtherapeutic levels of the drug during stable measurable disease results in tumor proliferation (19). However, the cessation of imatinib in the present cases may not necessarily be related to the recurrence of the tumors, since the tumors continued to respond to the treatment for as long as 43 months after the re-initiation of treatment. This observation supports our hypothesis that the probability of a secondary mutation increases with the duration of imatinib treatment, which most often is longer in GISTs with an exon 11 mutation than in those with exon 9 or 13 mutations or in wild-type GISTs.

Another significant finding in the present study involves the observation of a secondary mutation at the same site. Additionally, no more than one secondary KIT mutation was found in the same case. This is in conflict with the results of another recent study by Wardelmann (20), who found that 4 of 32 advanced progressive cases had more than one type of secondary KIT mutation. In the present study, 2 patients with wild-type KIT and PDGFRA genes developed primary progression while undergoing imatinib treatment, consistent with Sleijferet et al (21), who reported that tumors bearing a wild-type genotype in both KIT and PDGFRA always exhibit early resistance to imatinib. This indicates that, apart from CD117 being positive, the KIT/PDGFRA gene status detected in GISTs may be the most important event prior to imatinib therapy.

In the present investigation, in 4 of 10 imatinib-resistant GIST patients, mutation analysis of KIT from the primary GIST showed a point mutation or deletion in exon 11 of the $K I T$ gene. Analysis of the imatinib-refractory disease confirmed the presence of the primary exon 11 deletion and identified an acquired missense mutation at nucleotide position 2467 of exon 17. However, 8 patients with stable disease or partial remission were not found to carry a secondary mutation. No additional mutation was found in exons 9,11 or 13 of the KIT gene, or in exons 12 or 18 of the PDGFRA gene. To date, no untreated GISTs have been reported to have more than one mutation in KIT. Therefore, finding a second and new KIT mutation in closely monitored imatinib-resistant clones is convincing in vivo evidence of a causal relationship (22). Mutational analysis of these resected lesions identified additional acquired mutations in exon 17, indicating the likely pathogenetic mechanism of resistance.

In summary, we report a clinical and molecular study of acquired resistance to imatinib in gastrointestinal stromal tumors. We found that the exon-17 missense mutation T2467G, which tends to be a single amino acid substitution in the KIT kinase domain, appears to be the mechanism for late imatinib resistance in KIT exon 11-mutant GISTs. Our findings have implications for strategies to treat or avert imatinib resistance, and might be useful in the design of second-generation kinase inhibitors. 


\section{References}

1. Verweij J, Casali PG, Zalcberg J, et al: Progression-free survival in gastrointestinal stromal tumours with high-dose imatinib: randomised trial. Lancet 364: 1127-1134, 2004.

2. Van Glabbeke M, Verweij J, Casali PG, et al: Initial and late resistance to imatinib in advanced gastrointestinal stromal tumors are predicted by different prognostic factors: A European Organisation for Research and Treatment of Cancer-Italian Sarcoma Group-Australasian Gastrointestinal Trials Group Study. J Clin Oncol 23: 5795-5804, 2005.

3. Fletcher JA, Corless CL, Dimitrijevic S, et al: Mechanisms of resistance to imatinib mesylate in advanced gastrointestinal stromal tumor. Proc Am Soc Clin Oncol 22: abs. 3275815, 2003.

4. Debiec-Rychter M, Cools J, Dumez H, et al: Mechanisms of resistance to imatinib mesylate in gastrointestinal stromal tumors and activity of the PKC412 inhibitor against imatinib-resistant mutants. Gastroenterology 128: 270-279, 2005.

5. Therasse P, Arbuck SG, Eisenhauer EA, et al: New guidelines to evaluate the response to treatment in solid tumors: European Organization for Research and Treatment of Cancer, National Cancer Institute of the United States, National Cancer Institute of Canada. J Natl Cancer Inst 92: 205-216, 2000.

6. Pauls K, Merkelbach-Bruse S, Thal D, et al: PDGFR- and c-kit mutated gastrointestinal stromal tumors (GISTs) are characterized by distinctive histological and immunohistochemical features. Histopathology 46: 166-175, 2004.

7. Wardelmann E, Losen I, Hans V, et al: Deletion of Trp-557 and Lys-558 in the juxtamembrane domain of the c-kit protooncogene is associated with metastatic behavior of gastrointestinal stromal tumors. Int J Cancer 106: 887-895, 2003.

8. Wardelmann E, Hrychyk A, Merkelbach-Bruse S, et al: Association of platelet-derived growth factor receptor alpha mutations with gastric primary site and epithelioid or mixed cell morphology in gastrointestinal stromal tumors. J Mol Diagn 6: 197-204, 2004.

9. Kikuchi H, Yamashita K, Kawabata T, et al: Immunohistochemical and genetic features of gastric and metastatic liver gastrointestinal stromal tumors: Sequential analyses. Cancer Sci 97: 127-132, 2006.

10. Joensuu H, Roberts PJ, Sarlomo-Rikala M, et al: Effect of the tyrosine kinase inhibitor STI571 in a patient with a metastatic gastrointestinal stromal tumor. N Engl J Med 344: 1052-1056, 2001 .
11. Demetri GD, von Mehren M, Blanke CD, et al: Efficacy and safety of imatinib mesylate in advanced gastrointestinal stromal tumors. N Eng J Med 347: 472-480, 2002.

12. Heinrich MC, Corless CL, Blanke CD, et al: Molecular correlates of imatinib resistance in gastrointestinal stromal tumors. J Clin Oncol 24: 4764-4774, 2006.

13. Wakai T, Kanda T, Hirota $S$, et al: Late resistance to imatinib therapy in a metastatic gastrointestinal stromal tumour is associated with a second KIT mutation. Br J Cancer 90: 2059-2061, 2004.

14. Tamborini E, Bonadiman L, Greco A, et al: A new mutation in the KIT ATP pocket causes acquired resistance to imatinib in a gastrointestinal stromal tumor patient. Gastroenterology 127: 294-299, 2004.

15. Antonescu CR, Besmar P, Tianhua G, et al: Acquired resistance to imatinib in gastrointestinal stromal tumor occurs through secondary gene mutation. Clin Cancer Res 11: 4182-4190, 2005.

16. Mol CD, Dougan DR, Schneider TR, et al: Structural basis for the autoinhibition and STI-571 inhibition of c-Kit tyrosine kinase. J Biol Chem 279: 31655-31663, 2004.

17. Heinrich MC, Corless CL, Demetri GD, et al: Kinase mutations and imatinib response in patients with metastatic gastrointestinal stromal tumors. J Clin Oncol 21: 4342-4349, 2003.

18. Debiec-Rychter M, Dumez H, Judson I, et al: Use of c-kit/ PDGFRA mutational analysis to predict the clinical response to imatinib in patients with advanced gastrointestinal stromal tumours entered on phase I and II studies of the EORTC Soft Tissue and Bone Sarcoma Group. Eur J Cancer 40: 689-695, 2004.

19. Blay JY, Bonvalot S, Casali P, Choi H, Debiec-Richter M, Dei Tos AP, et al: Consensus meeting for the management of gastrointestinal stromal tumors. Report of the GIST Consensus Conference of 20-21 March 2004, under the auspices of ESMO. Ann Oncol 16: 566-578, 2005.

20. Wardelmann E, Merkelbach-Bruse S, Pauls K, et al: Polyclonal evolution of multiple secondary KIT mutations in gastrointestinal stromal tumors under treatment with imatinib mesylate. Clin Cancer Res 12: 1743-1749, 2006.

21. Sleijfer S, Wiemer E, Seynaeve C, et al: Improved insight into resistance mechanisms to imatinib in gastrointestinal stromal tumors: a basis for novel approaches and individualization of treatment. Oncologist 12: 719-726, 2007.

22. Chen LL, Trent JC, Wu EF, et al: A missense mutation in KIT kinase domain 1 correlates with imatinib resistance in gastrointestinal stromal tumors. Cancer Res 64: 5913-5919, 2004. 